Revista Luna Azul - MACROFLORA Y MACROFAUNA ASOCIADAS AL CORDÓ... Página 1 de 17

\title{
MACROFLORA Y MACROFAUNA ASOCIADAS AL CORDÓN ARRECIFAL DE LITTLE REEF (ISLA DE SAN ANDRÉS, COLOMBIA)
}

\author{
Ingrid García-Hansen \\ Western Maneuver Area 2603 \\ Lower Gainesville Rd. Stennis \\ Space Center, MS 39529-0001. USA \\ Correo electrónico: igarciahansen@hotmail.com \\ Ricardo Álvarez-León \\ Fundación Maguaré \\ Manizales (Caldas), Colombia \\ Correo electrónico: alvarez_leon@hotmail.com
}

Manizales, 2007-09-15 (Rev. 2007-11-10)

\section{RESUMEN}

Jornadas de observación con equipo autónomo de buceo, entre 1 y 20 m de profundidad, desde octubre de 1994 hasta enero de 1995 para un total de 75 horas, permitieron reconocer con ayuda de guías sumergibles y tablas acrílicas la macroflora y macrofauna representativa del arrecife, nunca antes registradas. El inventario incluye 7 taxa, 46 familias, 58 géneros y 78 especies. Entre los taxa registrados se presentan algas, fanerógamas, celenterados, poliquetos, equinodermos, crustáceos y peces, a los cuales se les asignó un nivel de abundancia según el grado de aparición de cada especie, de acuerdo al lugar que ocupan en la columna de agua y a lo largo de los transectos lineales realizados (1 a 10 - 20 m). Los resultados del trabajo coinciden en cuanto a la variedad y diversidad de los grupos y especies, encontrados en el resto de arrecifes coralinos del Caribe colombiano. Sin embargo, se encontraron 23 nuevos registros (2 celenterados, 2 poliquetos, 1 equinodermo, 2 crustáceos, 15 peces), para la Isla de San Andrés (Colombia).

\section{PALABRAS CLAVE}

Flora, Fauna, Nuevos Registros, Little Reef, San Andrés Isla, Caribe, Colombia. MACROFLORA AND MICROFAUNA ASSOCIATED TO THE BARRIER REEF OF
LITTLE REEF (SAN ANDRÉS ISLAND, COLOMBIA)

\begin{abstract}
Observation journeys with an autonomous diving team, between 1 and $20 \mathrm{~m}$ of depth, from October 1994 up to January 1995 (75 hours total), permitted the recognition, with the help of submergible guides and acrylic charts, of the representative macroflora and macrofauna of the reef never before registered. The inventory includes 7 taxa, 46 families, 58 genera and 78 species. Among the registered taxa algae, fanerogams, celenterates, poliquets, equinoderms, crustaceans and fish are included, which were assigned a level of abundance according to the degree of appearance of each species, according to the place they occupied in the water column and along the transect lineal replicates (1 at $10-20 \mathrm{~m}$ ). The results of the work coincide in the variety and diversity of
\end{abstract}


Revista Luna Azul - MACROFLORA Y MACROFAUNA ASOCIADAS AL CORDÓ... Página 2 de 17

the groups and species, found in the rest of coralline reefs of the Colombian Caribbean. However, 23 new registrations were found (2 celenterates, 2 poliquets, 1 equinoderm, 2 crustaceans, 15 fish), for the San Andrés Island (Colombia).

\section{KEY WORDS}

Flora, fauna, new reports, Little Reef, San Andres Island, Caribbean, Colombia.

\section{INTRODUCCIÓN}

Entre los estudios realizados en las áreas arrecifales de la Isla de San Andrés se encuentran los trabajos de Geister $(1973,1975)$ el cual hace una descripción de los arrecifes de la Isla de San Andrés, teniendo en cuenta los rasgos topográficos, sedimentológicos y la distribución de la fauna bentónica en los sectores noreste y este de la plataforma submarina de la isla. Contreras (1983) y Borrero y González (1983), entre otros, tratan aspectos relacionados con los agentes de polución causantes del deterioro de los ecosistemas marinos en la isla entre ellos los arrecifes de coral; Prahl y Erhardt (1985) describen los corales y formaciones coralinas de los mares colombianos. Díaz-Merlano et al. (1995) por medio de la utilización de transectos y la observación, brindan un diagnóstico del estado actual de los arrecifes coralinos de la Isla de San Andrés y proponen perspectivas para su conservación. Otros autores como BarrigaBonilla et al. (1969); Gómez-Niño y Victoria-Daza (1980, 1986) y Melendro (1990) contribuyeron al conocimiento de la ecología, flora, fauna y pesca de la isla de San Andrés.

El interés por estudiar esta zona surgió del deterioro que presenta el coral en el área, la abundancia de peces herbívoros (Scarus coeruleus, S. guacamaia, Sparisoma viride) y organismos herodadores, y la proliferación de algas (Caulerpa racemosa y Dictyota sp.) que actúan como indicadores biológicos y dan una idea de la presencia de altos niveles de materia orgánica en la columna de agua; observaciones similares fueron realizadas por Díaz-Merlano et al. (1995). Esta situación resulta benéfica para las citadas algas y obviamente para los herbívoros, pues han logrado ganar bastante terreno en perjuicio de los corales y de la gran diversidad de organismos que habitan Little Reef.

Con la idea de ofrecer una base que permita proteger este importante sistema arrecifal, se realizó el inventario de las especies presentes en el cordón arrecifal de Little Reef. Se ofrece además una descripción breve de cada una de ellas, que incluye nombre vernacular, características diferenciales y conspicuas para su identificación en el campo, hábitat, asociaciones, importancia, abundancia y aprovechamiento, cuando existe en la zona.

\section{Área de Estudio}

Los arrecifes coralinos del archipiélago de San Andrés, Providencia y Santa Catalina, son ecosistemas muy complejos y productivos que sirven como lugares de alimentación, crianza y refugio de una gran variedad de organismos, tanto invertebrados como vertebrados, especialmente diversos en formas y colores, constituyéndose además en lugares de gran belleza paisajística.

En general, los arrecifes forman la base de todos los atolones, en aguas tibias y poco profundas, ricas en calcio para la construcción de sus exoesqueletos. Es raro que los atolones presenten una configuración de óvalo ideal o contorno circular con los que a menudo se les ilustra. En realidad, reflejan los contornos de sus cimientos submarinos, pudiendo hallarse en todas las formas imaginadas y generalmente con una longitud aproximadamente de dos veces la anchura (Boolotian, 1975).

San Andrés es una isla oceánica localizada en el Caribe occidental, entre los $12^{\circ} 28^{\prime}$ y $12^{\circ} 36^{\prime} \mathrm{N}$ y los $81^{\circ} 40^{\prime}$ y $81^{\circ} 44^{\prime} \mathrm{W}$. La historia geológica de la isla es muy interesante y dinámica, pues se originó como un atolón durante el Terciario, entre el Oligoceno y el 
Revista Luna Azul - MACROFLORA Y MACROFAUNA ASOCIADAS AL CORDÓ... Página 3 de 17

Revista Luna Azul

No. 25, Julio - Diciembre 2007

Mioceno. Al crecer las formaciones coralinas como arrecifes costeros en las orillas del cono volcánico, se formó un anillo coralino de borde que fue creciendo verticalmente a medida que el cono volcánico se hundía, pero de tal forma que el anillo podía crecer y mantenerse cerca de la superficie. La laguna central del atolón se fue llenando con sedimentos y ceniza volcánica transportada por los vientos. Durante el Plioceno e inicios del Pleistoceno éste atolón fue emergiendo y exponiéndose hasta formar una isla coralina (Geister, 1973).

La Isla de San Andrés se caracteriza por presentar tres tipos de formaciones coralinas: arrecifes de borde o costeros, parches coralinos y arrecifes de barrera que se encuentran mezclados hacia la zona noreste de la isla.

\section{Cordón Arrecifal de Little Reef}

El arrecife de Little Reef se encuentra localizado al extremo noreste de la Isla de San Andrés, a unos $100 \mathrm{~m}$ de la línea costera y en sentido paralelo a ésta (Fig. 1). Presenta una extensión aproximada de 1300 m de longitud (CORALINA, 1997a) y hace parte de un complejo de arrecifes conformados por 6 pequeños parches de coral ubicados dentro de la laguna arrecifal. La zona de la laguna en donde se desarrolla este arrecife, se caracteriza por estar al abrigo del oleaje y por el predominio de ambientes sedimentarios, parcialmente vegetados por fanerógamas marinas y algas, principalmente algas costrosas y dictiotáceas frondosas (Díaz-Merlano et al., 1995).

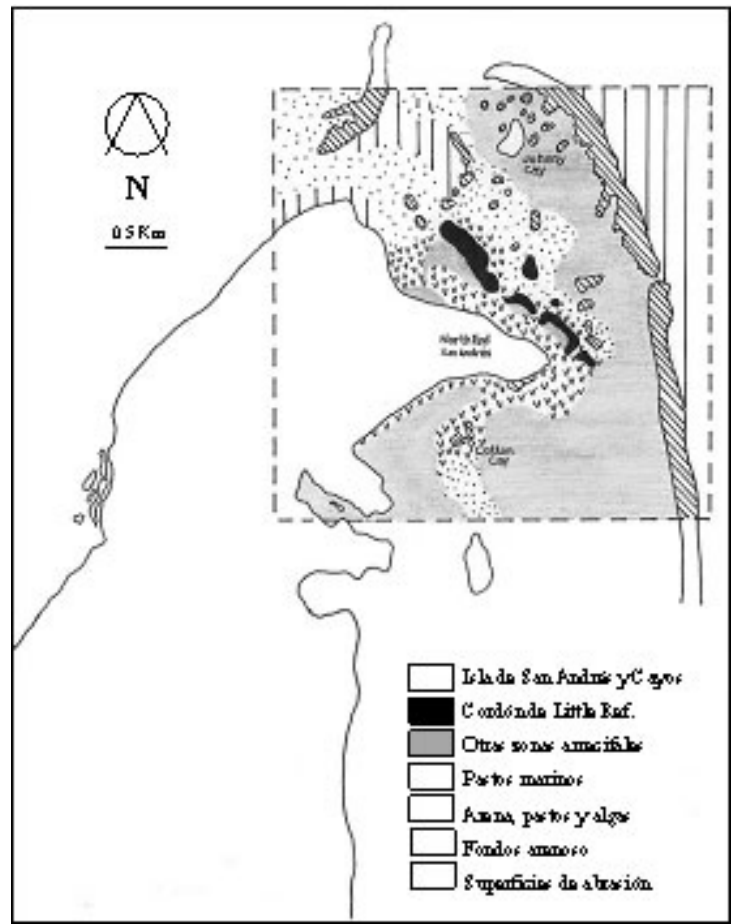

Fig. 1. Ubicación del cordón arrecifal de Little Reef, noreste de la Isla de San Andrés. Tomado de Geister (1976).

Estos arrecifes alcanzan la superficie desde unos 4 a $5 \mathrm{~m}$ de profundidad sobre el fondo de la laguna y se consideran como una barrera secundaria no continua. Según Díaz-Merlano et al. (1995) el aspecto general de estos parches es muy similar, presentando una cobertura coralina entre 20 y $45 \%$ del sustrato duro, y la dominancia de Millepora complanata, Acropora palmata, Porites astreoides y Diploria strigosa. Por otro lado, detrás de la cresta de estos arrecifes lagunares, se encuentra por lo general un plano de coral muerto y escombros coralinos con cabezas aisladas de D. strigosa, continuada por una zona dominada por D. clivosa y P. astreoides, y que a su vez continua con una cresta posterior de A. palmata. Frecuentemente, el erizo Tripneustes ventricosus muy abundante en las praderas de Thalassia, invade el plano arrecifal en altas densidades (hasta 8 ind./m²) (Díaz-Merlano et al., 1995). 
En esta zona presenta una alta mortalidad de coral la cual podría deberse a factores biológicos tales como enfermedades, condiciones medioambientales adversas, depredación por parte de organismos herodadores (peces, gastrópodos, poliquetos, equinodermos), proliferación de algas, entre otros. Según Geister (1976) la destrucción generalizada de los arrecifes conformados por Acropora cervicornis en el sector occidental de Little Reef y en menor medida también sobre los de A. palmata se debió al paso del huracán Irene en 1971. Situación que se vio empeorada posteriormente con el paso del huracán Joan en 1988, el cual de igual manera afectaría de forma crítica la situación de estos arrecifes (Melendro, 1990). Por otro lado, existen un variado número de causas antropogénicas que han afectado a las comunidades coralinas en la Isla de San Andrés y que a su vez han impedido la recuperación y mantenimiento de este ecosistema. Entre las causas se encuentran: la sobre-explotación de recursos renovables; la destrucción del hábitat y sedimentación debida a construcciones litorales, dragados y extracción de roca coralina y arena; contaminación por la introducción de sólidos y líquidos derivados de distintas actividades; y la destrucción del hábitat por daño accidental en usos recreacionales y turísticos (Díaz-Merlano et al., 1995).

\section{MATERIALES Y MÉTODOS}

Para la realización de este estudio, el área de Little Reef fue considerada como un solo complejo arrecifal; por lo que se presentan los resultados en general para toda la zona y sin tener en cuenta cada parche por separado. Se realizaron 75 horas de observación con equipo autónomo de buceo, entre 1 y 20 m de profundidad, desde octubre de 1994 hasta enero de 1995.

Los organismos fueron reconocidos por medio de observaciones realizadas en las áreas y se establecieron categorías para calificar su abundancia, de acuerdo al lugar que ocupan las especies en la columna de agua y a lo largo de los transectos lineales realizados (1 a 10 - 20 m). Como material de campo se utilizó la guía de Stokes (1980) y las guías sumergibles de Boer et al.(1973), Pfluger y Smiley (1974), Colin (1978), Greenberg (1986), las tablas acrílicas ilustradas, así como la colecta de los organismos cuando no era factible identificarlos in situ, durante las faenas de buceo.

En los resultados se presenta una síntesis de las especies determinadas, anotando todos los datos de campo y laboratorio, ordenadas bajo el nombre vernacular de cada grupo y de acuerdo al nombre en latín de cada especie. En la tabla 1 se ordenaron de acuerdo a su categoría taxonómica: Clase, Familias, Géneros y Especies, así como especificando su hábitat, abundancia y registros previos para la Isla de San Andrés (Colombia), de todos los organismos del necton y bentos.

\section{RESULTADOS}

De acuerdo a las observaciones realizadas se encontraron y determinaron 7 taxa, 46 familias, 58 géneros y 78 especies (Tabla 1), dentro de la macroflora y macrofauna más característica del área.

\section{Algas Verdes}

Caulerpa racemosa (alga verde / green algae): presenta estructuras ovaladas, cilíndrico-claviforme con brazos erectos y largos. Habita en aguas muy claras del arrecife y puede actuar como indicador biológico o de contaminación orgánica. Esta especie se destaca en el área de estudio por ser muy abundante y por la notoria competencia que ha establecido sobre las especies de coral, habiendo ganado terreno sobre todo en las zonas habitadas por cabezas coralinas, deditos de coral y escombros de coral muerto.

Halimeda opuntia (alga verde calcárea / green calcareus algae): su estructura es a manera de hojuelas calcáreas de color verde intenso. Ocupan grandes extensiones del arrecife, sirviendo como refugio a organismos pequeños. Se observa de manera abundante en la zona de Little Reef, sobre todo rodeando el arrecife y asentándose sobre fondos arenosos.

Ventricaria ventricosa (alga globosa / spherical algae): se trata de una célula expandida que alcanza un máximo de $5 \mathrm{~cm}$ de diámetro. Crece sobre las rocas, cabezas de coral 
Revista Luna Azul - MACROFLORA Y MACROFAUNA ASOCIADAS AL CORDÓ... Página 5 de 17

y corales de dedo. Puede desarrollarse en colonias masivas sobre el arrecife o solitarias y aisladas. Son de coloración verde oscura con tonos marrones y a veces presenta como epífitas, algas rojas, coralináceas (costrosas). En el área de Little Reef se encuentra habitando sobre cabezas de coral y coral muerto, y es muy poco abundante.

\section{Algas Pardas}

Dictyota spp. (alga parda / iridaceous algae): crece de manera abundante sobre el arrecife y los fondos rocosos; libera sustancias químicas para competir por su espacio y defenderse de sus depredadores aunque, a pesar, de esto hay peces que la consumen. Sus hojas son bifurcadas y de color verde o marrón oro iridiscente. Es muy abundante en el área de estudio y al igual que C. racemosa ha invadido amplias zonas del arrecife. Barriga-Bonilla et al. (1969) registró para la Isla de San Andrés a D. ciliolata y $D$. dentata; Kapraum (1972) a D. bartauresii; y Schnetter (1978) a D. bartauresii, $D$. cervicornis, $D$. ciliolata, $D$. dentata, $D$. indica y $D$. linearis.

\section{Fanerógamas}

Syringodium filiforme (hierba marina / manatee grass): su hoja es delgada, tiene forma de espagueti y es de coloración verde claro. Es poco abundante en el área y se encuentra formando pequeños parches o entremezclada con $T$. testudinum.

Thalassia testudinum (pasto marino / turtle grass): su hoja es ancha y de color verde oscuro, pudiendo alcanzar una longitud de $30 \mathrm{~cm}$. Se observa de manera muy abundante en el área, extendiéndose desde la costa hasta rodear prácticamente los parches que conforman el arrecife de Little Reef (Fig. 1).

\section{Anémonas}

Condylactis gigantea (anémona verde / greater green anemone): es una de las más comunes en el arrecife. Posee una coloración muy vistosa con tentáculos verdes de punta color violeta, aunque dependiendo de la profundidad estas tonalidades pueden cambiar y ser más claras o casi blancas cuando se encuentra en zonas con muy poco sol. Su nombre científico se debe a que posee los tentáculos más largos de esta familia. Son poco abundantes en el área de estudio y se encuentran asentadas sobre el arrecife.

\section{Corales Córneos}

Gorgonia ventalina (abanico común / common sea fan): es una especie muy poco abundante o esporádica en el área, presentando una coloración violeta oscuro y tamaños pequeños que van entre 30 y $70 \mathrm{~cm}$ de longitud. Los individuos observados se encuentran aislados unos de otros, habitando sobre substratos duros del arrecife.

\section{Octocorales}

Plexaura cf. homomala (dedos marinos / corky sea fingers): presenta pólipos largos y usualmente extendidos, los cuales contrae cuando es perturbada. Su coloración va de amarillo quemado a marrón y ocres. Son poco abundantes en el área de estudio.

\section{Falsos corales}

Millepora complanata (coral de fuego / leaf stinging coral): la colonia está formada por placas verticales, delgadas y lisas unidas por sus bases, de color amarillo quemado o amarillo marrón. Es sin lugar a dudas el componente fundamental de las crestas coralinas y zonas expuestas de los espolones arrecifales. Su nombre se debe a las quemaduras que produce al contacto con la piel del hombre. Se observa de manera muy abundante, sobre todo en los parches ubicados al centro y este de Little Reef.

\section{Corales pétreos}

Acropora palmata (coral cuerno de alce / elkhorn coral): presenta colonias con ramificaciones foliares anchas y aplanadas, de color habano o marrón suave. Esta especie se encuentra habitando aguas someras expuestas al oleaje, generalmente de 
0.5 a $15 \mathrm{~m}$ de profundidad. Se observa de manera poco abundante al norte, centro y este de Little Reef, con apariciones de nuevos individuos y presentándose una mortalidad casi total de la especie al oeste del arrecife.

Agaricia agaricites (coral galleta / leaf coral): forma colonias planas y gruesas o con lóbulos, alcanzando de 1 a 3 m de diámetro. De color marrón oscuro a marrón púrpura. Es un coral típico de aguas someras y relativamente protegidas, en las zonas expuestas se asocia a otros corales. Es poco abundante en el área y especialmente se encuentra colonizando las zonas ocupadas por coral muerto.

Diploria clivosa (coral cerebro / knobby brain coral): colonias formadas por esteras o meandros planos de forma irregular. Alcanzan diámetros superiores a $1 \mathrm{~m}$. Su apariencia externa no es hemisférica como en el caso de $D$. strigosa sino conformada por montículos de tamaño variado. Presenta una coloración marrón que va de tonos claros a oscuros. Es poco abundante en el área, observándose con mayor frecuencia al noroeste de Little Reef.

D. strigosa (coral cerebro / smooth brain coral): colonias en forma de meandro regularmente espaciadas, que pueden alcanzar tallas superiores a los $2 \mathrm{~m}$ de diámetro. Su coloración es amarilla o verde marrón con tonalidades azulosas. Habita desde 0.2 a $40 \mathrm{~m}$ de profundidad encontrándose generalmente sobre el borde de las barreras arrecifales. Se presenta como una especie muy abundante en la zona de estudio.

Meandrina meandrites (coral meandro / butterprint brain coral): como su nombre lo indica sus pólipos se ubican a manera de meandros, formando una estructura similar a la del coral cerebro. Alcanza un diámetro de más de $1 \mathrm{~m}$. De coloración marrón o amarilla. Puede encontrarse a una profundidad de 5 a $10 \mathrm{~m}$ cuando se encuentra habitando los arrecifes, pero cuando forma colonias aisladas únicamente se le encuentra entre los 20 a 40 m de profundidad. Es una especie muy poco abundante o esporádica en el área.

Montastrea annularis (coral de pólipos protuberantes / mountainous star coral): forma colonias masivas, hemisféricas que pueden adoptar incluso formas de crecimiento plano.

Porites astreoides (coral de montículos / mustard hill coral): las colonias crecen en forma de montículos o lóbulos los cuales pueden presentar baches o ser aplanados. Estos montículos se encuentran poco espaciados entre si, característica que lo diferencia de Madracis decactis. El color varía de amarillo quemado a marrón. Algunas veces las colonias crecen incrustadas en coral muerto. Es una especie abundante en el área, observándose entre la costa y los parches que conforman este arrecife, sobre todo en las zonas más someras.

P. porites (coral de dedos / club finger coral): son colonias digitiformes y ramosas hasta de $50 \mathrm{~cm}$ de altura, pudiéndose extender en tapetes de varios metros. Las ramificaciones pueden variar desde los $5 \mathrm{~mm}$ de diámetro hasta ramificaciones masivas de más de $25 \mathrm{~mm}$. Su coloración es amarilla, gris y marrón. Su forma varía dependiendo de la profundidad. Generalmente se encuentra en aguas someras de lagunas arrecifales. Al igual que la anterior se observa de manera abundante en el área de estudio.

Siderastrea siderea (coral estrella / smooth starlet coral): de forma globosa con pólipos a manera de estrella, presenta colonias que alcanzan $2 \mathrm{~m}$ de diámetro y una coloración marrón con tonos rojizos. Se encuentra comúnmente entre los 0.5 hasta $2 \mathrm{~m}$ de profundidad, aunque puede habitar hasta $70 \mathrm{~m}$ de profundidad, formando arrecifes de parche y hacia el borde interno del arrecife. Esta especie se observa especialmente al oeste de Little Reef y es poco abundante.

\section{Poliquetos}

Sabellastarte sp. (gusano plumero / feather worm): habitan dentro de un tubo largo de color marrón y poseen unas prolongaciones a manera de penacho marrón y blanco, los cuales contraen al presentarse disturbios en el medio. La función principal de estos penachos es crear corrientes de agua para atraer el alimento. Son muy abundantes en 
Revista Luna Azul - MACROFLORA Y MACROFAUNA ASOCIADAS AL CORDÓ... Página 7 de 17

el área de estudio y se encuentran habitando principalmente las cabezas de coral.

Spirobranchus sp. (gusano espiral / spiral worm): sus tentáculos se prolongan a manera de espiral. Pueden llegar a formar grandes colonias. Su coloración puede ser roja, naranja, blanco o la combinación de estos tres. Se caracteriza por la gran velocidad con la cual contrae sus tentáculos al verse amenazado. Al igual que los anteriores habitan en cabezas de coral y son muy abundantes en el área.

\section{Holoturidos}

Isostichopus sp. (pepino de mar común / common sea cucumber): es de coloración amarilla con manchas marrón. Es una de las especies más comunes en el área. Para defenderse de sus enemigos produce una sustancia tóxica con la cual evita ser predado. Fueron observados de manera abundante habitando sobre fondos arenosos cerca del arrecife y dentro de las praderas de Thalassia.

\section{Erizos}

Diadema antillarum (erizo negro / long spined sea urchin): alcanza un diámetro máximo de $10 \mathrm{~cm}$ y las espinas una longitud de 30 a $40 \mathrm{~cm}$, las cuales pueden ser negras o blancas. Estas espinas contienen sustancias tóxicas que pueden causar severas infecciones en el hombre. Esta especie habita los pastos marinos, los arrecifes rocosos, las áreas de manglar y los fondos blandos de aguas claras. Esta especie se observó de manera abundante en la zona, habitando los fondos duros del arrecife.

Echinometra lucunter (erizo rojo / red sea urchin): esta especie se caracteriza por habitar en cuevas ubicadas en litorales rocosos o en arrecifes costeros, posee espinas gruesas de una longitud entre 5 y $10 \mathrm{~cm}$. Sólo se observaron algunos individuos al centro y este del arrecife.

Tripneustes ventricosus (erizo blanco / white sea urchin): se caracteriza por poseer espinas cortas que alcanzan una longitud de 2 a $4 \mathrm{~cm}$, de coloración blanquecina a gris muy pálido. La especie se encuentra distribuida de manera muy abundante en el área sobre las praderas de $T$. testudinum, especialmente al noroeste de Little Reef.

\section{Cangrejos}

Stenopus hispidus (camarón elegante o payaso / red band coral shrimp): marinos de aguas tropicales, habitan hasta los $15 \mathrm{~m}$ de profundidad, son de hábitos nocturnos, camuflándose en el día entre los corales o las piedras. Son limpiadores de parásitos de peces para lo cual usan sus largas tenazas, poseen antenas muy sensitivas. Su cuerpo está cubierto por bandas de color rojo y blanco, haciéndolo muy llamativo. Son poco abundantes en el área de Little Reef y se encuentran habitando cavernas arrecifales.

Stenorhynchus seticornis (cangrejo flecha o araña / spider crab): es uno de los cangrejos más representativos de los arrecifes de coral de las aguas tropicales del Mar Caribe; por lo general habitan hasta los $180 \mathrm{~m}$ de profundidad. Sus patas son muy largas con relación al cuerpo y sus ojos son de color rojo encontrándose opuestos a ambos lados de la cabeza. Su coloración puede ser blanca, marrón u ocre. Muchas veces se encuentran asociados a esponjas y anémonas. Son poco abundantes en el área de estudio, en donde se observaron tan sólo asociados con anémonas.

\section{Langostas}

Panulirus argus (langosta espinosa / spiny lobster): habitan en arrecifes coralinos y fondos rocosos. La coloración del caparazón es naranja fuerte y como su nombre lo indica presentan protuberancias a manera de espinas en la región anterior del caparazón. Alcanzan una talla de $66 \mathrm{~cm}$, aunque las tallas más frecuentes están entre 15 a $25 \mathrm{~cm}$. Se presentó como una especie esporádica en la zona y sólo se le observó camuflada dentro de grietas en el arrecife.

\section{Rayas}

Dasyatis sabina (raya del Atlántico / southern stingray): su coloración va de tonos grises 
Revista Luna Azul - MACROFLORA Y MACROFAUNA ASOCIADAS AL CORDÓ... Página 8 de 17

a marrón y tiene una o más espinas fuertes en la cola. Son poco abundantes en el área, sobre fondos arenosos y son difícilmente visibles ya que se entierran en la arena.

\section{Tiburones}

Ginglymostoma cirratum (tiburón nodriza / nurse shark): es de color amarillo quemado o marrón y su piel se utiliza en la fabricación de lijas. Las aletas caudales y pectorales son bastante largas con relación al cuerpo. Se le observa en el área de Little Reef como un individuo solitario y esporádico, desplazándose sobre el fondo arenoso o escondido bajo el arrecife.

\section{Peces Óseos}

Abudefduf saxatilis (sargento mayor / sergeant major): se caracteriza por sus bandas negras y amarillas a través del cuerpo. Presentan cuidado parental y cuando el macho cuida los huevos su coloración se oscurece. Su talla es de $18 \mathrm{~cm}$ de longitud total. Se le observa de manera muy abundante en la zona, merodeando el arrecife y alimentándose de animales y plantas.

Acanthurus chirurgus (cirujano marrón / doctorfish): es de color marrón con líneas verticales un poco más oscuras que atraviesan el cuerpo. El borde de las aletas dorsales, anales y pélvicas es azul. La espina de la aleta caudal esta rodeada de un margen negro y uno azul. Su talla es de $30 \mathrm{~cm}$ de longitud total. Son muy abundantes en el área de Little Reef y se desplazan en pequeños cardúmenes rondando por el arrecife a cualquier profundidad.

A. coeruleus (cirujano azul / blue tang): de coloración azul celeste o grisáceo en todo el cuerpo y estrías grises oscuras en los costados y las aletas. La espina del pedúnculo caudal esta rodeada por un margen blanco. Los juveniles son totalmente amarillos y se van tornando azules a medida que crecen. Su tamaño supera los $35 \mathrm{~cm}$ de longitud total. Es una especie muy abundante en el área y al igual que la anterior anda en cardúmenes merodeando el arrecife.

Bodianus rufus (loro gallo español / spanish hogfish): la porción dorso-anterior de su cuerpo es azul, violeta o rojo dependiendo de la profundidad y, el resto, es amarillo. En estado juvenil es de hábitos limpiadores. Alcanza $30 \mathrm{~cm}$ de longitud total. Es medianamente abundante en Little Reef y se le observa rondando por el arrecife.

Caranx hippos (jurel común o caballa / crevalle jack): su cuerpo es de color plateado azuloso y las aletas caudales, anales y pélvicas presentan una coloración amarillosa. Es la especie más veloz dentro del grupo de los jureles. Pueden alcanzar tallas superiores a $1 \mathrm{~m}$ de longitud total. Se observan en la zona nadando velozmente al rededor del arrecife y de manera esporádica.

C. ruber (jurel de barra azul / bar jack): se caracterizan por tener una línea azul oscura en el dorso y su cuerpo es de color plateado. Dentro del área generalmente se observan en parejas o en pequeños grupos, siendo poco abundantes. Son muy activos y nadan a gran velocidad al rededor del arrecife.

Chaetodon capistratus (mariposa cuatro ojos / foureye butterflyfish): posee un ocelo negro bordeado por un halo blanco hacia la región caudal y una barra negra que atraviesa el ojo, se cree que este patrón de coloración es una estrategia que utiliza esta especie para despistar a sus enemigos, los cuales se dirigen a la región caudal y no a la cabeza al momento del ataque. Alcanzan una longitud total de $12 \mathrm{~cm}$. Son abundantes dentro del arrecife de Little Reef y se observan formando parejas o en pequeños grupos.

C. ocellatus (mariposa de océlo / spotfin butterflyfish): presenta cuatro ocelos de color oscuro hacia la región posterior, un par a cada lado del cuerpo, siendo el resto de este de color amarillo claro. Su tamaño es de $18 \mathrm{~cm}$ en promedio. Siempre se les observa formando parejas y son abundantes en el área de estudio.

C. striatus (mariposa rayada / banded butterflyfish): con dos bandas negras que atraviesan el cuerpo del dorso al vientre y otra más delgada que atraviesa el ojo. Su 
Revista Luna Azul - MACROFLORA Y MACROFAUNA ASOCIADAS AL CORDÓ... Página 9 de 17

talla máxima es alrededor de $14 \mathrm{~cm}$. Son abundantes dentro del arrecife y andan en parejas o en grupos.

Chromis cyaneus (cromis azul / blue chromis): de color azul iridiscente y con una línea negra en el dorso que va desde el hocico hasta la punta de la aleta caudal. De igual manera la aleta dorsal presenta un borde negro. Alcanza una longitud total de $12 \mathrm{~cm}$. En el área de estudio se le observó de manera muy abundante en grandes cardúmenes sobre el arrecife.

Diodon hystrix (pez puerco o erizo / porcupinefish): el dorso es color verde oliva a marrón, con numerosos puntos negros y vientre blanco. Posee espinas cortas y gruesas que se levantan como defensa en el momento de ser perturbados. Su talla promedio es de $50 \mathrm{~cm}$ de longitud total, sin embargo puede alcanzar $1.20 \mathrm{~m}$. Es poco abundante en al área y se le encuentra algunas veces cerca del fondo arenoso, aunque por lo general se esconde en grietas o cavernas dentro del arrecife.

Epinephelus adcensionis (mero roca / rock hind): se caracteriza por tener puntos marrones oscuros en todo el cuerpo, además de dos manchas oscuras en la región dorsal en la base de la aleta y una más pequeña sobre el pedúnculo caudal detrás de la aleta dorsal. Su longitud total es de $50 \mathrm{~cm}$. Estos individuos poco abundantes en la zona de Little Reef, fueron además difícilmente observados ya que se encontraban por lo general dentro de grietas en el arrecife.

E. guttatus (mero rojo / red hind): presenta márgenes oscuros al borde de las aletas dorsal, anal y caudal, y puntos rojos discontinuos sobre todo el cuerpo, el cual a su vez también es rojizo. Alcanza $50 \mathrm{~cm}$ de longitud total. Es abundante en el área de Little Reef.

Fistularia tabacaria (trompetero / bluespotted cornetfish): puede alcanzar $2 \mathrm{~m}$ de longitud total y su cuerpo es alargado y deprimido. Con colores que van desde rojizos y anaranjados hasta azules o con manchas verdes. Es una especie poco abundante en la zona, caracterizándose por ser un habitante solitario de media agua, la cual ronda sobre praderas de Thalassia y el arrecife.

Gobiosoma genie (gobio limpiador / cleaning goby): se caracteriza por la presencia de una mancha amarilla en forma de triángulo sobre la cabeza, la cual se va difuminando sobre una línea blancuzca que atraviesa el cuerpo horizontalmente. Es de hábitos limpiadores. Es esporádico en el área de estudio y habita sobre cabezas de coral.

G. oceanops (gobio azul / neon goby): presenta bandas longitudinales azul oscuro y negras sobre el cuerpo, con aletas azul claro casi transparentes. Al igual que la especie anterior es de hábitos limpiadores. En el área de Little Reef se presenta como una especie poco abundante y se le observa habitando principalmente sobre las cabezas de coral cerebro. Puede encontrarse solitario o en parejas.

Haemulon aurolineatum (ronco gris / tomtate grunt): se caracteriza por la presencia de una mancha oscura a cada lado del pedúnculo caudal. El cuerpo es de color gris plateado y presenta una talla superior a los $12 \mathrm{~cm}$. Es muy abundante en Little Reef.

H. chysargyreum (ronco bocapequeña / smallmouth grunt): presenta 6 líneas amarillas claramente visibles, pero que al intercalarse y hacer contraste con el resto del cuerpo se difuminan dándole al individuo una apariencia completamente plateada. Todas las aletas son de color amarillo. Su talla máxima es de $12 \mathrm{~cm}$. Se observa de manera muy abundante en el arrecife formando cardúmenes.

H. flavolineatum (ronco francés / french grunt): su color es amarillo brillante con líneas azulosas de patrones ondeantes que atraviesan el cuerpo del individuo. Las aletas son amarillas. Esta especie es muy abundante en el área de estudio y forma pequeños grupos.

H. plumieri (ronco blanco / white grunt): posee líneas azules claramente visibles que atraviesan la cabeza y que siguen a lo largo del cuerpo de forma discontinua a manera de escamas intercaladas azules y amarillas. Su talla máxima es de $20 \mathrm{~cm}$ y es una especie abundante en el área. 
H. sciurus (ronco amarillo / bluestriped grunt): coloración amarillo bronce en todo el cuerpo, con líneas azules horizontales. Alcanza una longitud total de $30 \mathrm{~cm}$, siendo la especie de mayor tamaño dentro del grupo de los roncos. Es abundante en la zona de estudio y se le puede observar tanto en pequeños grupos como solitario.

Holocanthus ciliaris (angel reina / queen angelfish): esta especie es de gran colorido y se caracteriza por tener un ocelo azul iridiscente en la cabeza en su estado adulto. El juvenil presenta tres barras azules sobre el cuerpo, dos sobre la cabeza y una barra oscura alrededor del ojo. El adulto sobrepasa los $25 \mathrm{~cm}$ de longitud total, existiendo registros de individuos que han alcanzado los $40 \mathrm{~cm}$. Son poco abundantes en Little Reef y se les observa solitarios, merodeando en el arrecife.

$H$. tricolor (ángel roca o belleza de las rocas / rock beauty): en estado adulto la parte anterior y la aleta caudal son amarillo fuerte y el resto del cuerpo es negro. El borde anterior de la aleta anal y la branquia son anaranjados. Los juveniles son amarillos con tan sólo una mancha negra, la cual se va expandiendo a medida que el individuo va creciendo. Alcanzan $25 \mathrm{~cm}$ de longitud total. Son poco abundantes en el área.

Holocentrus adscensionis (carajuelo común o gallito / squirrelfish): presenta una coloración rojiza plateada alternando con líneas blancas. Posee la aleta dorsal de color amarillo y alcanza $60 \mathrm{~cm}$ de longitud total Se presenta de manera abundante dentro del área de estudio.

$H$. rufus (carajuelo soldado / longspine squirrelfish): se caracteriza por tener puntos blancos en la aleta dorsal y alcanzar $35 \mathrm{~cm}$ de longitud total. Es una especie medianamente abundante en el área.

Hypoplectrus puella (vaca de barras o hamlet de barras / barred hamlet): su cuerpo es de color marrón oscuro con barras castaño claro en la parte posterior y azules en la parte anterior, alcanzan no más de $15 \mathrm{~cm}$ de longitud total. Es poco abundante en el área, observándose en aquellas zonas en donde se presenta un alto crecimiento de coral, dentro del cual busca esconderse y protegerse. Es una especie poco activa.

Mulloidichthys martinicus (chivo / yellow goatfish): se caracteriza por tener una barra longitudinal amarilla que va desde el ojo hasta cubrir la aleta caudal. Mide de 28 a 48 $\mathrm{cm}$ de longitud total en promedio. Es abundante en el área de Little Reef y se observa escarbando los fondos arenosos al rededor del arrecife. En algunas ocasiones se le encontró en las praderas de Thalassia.

Mycrospathodon chrysurus (pez joya / yellowtail damselfish): en estado juvenil presenta puntos azul fluorescente y en estado adulto estos puntos van desapareciendo hasta presentar un color marrón oscuro casi uniforme, la aleta caudal es amarilla. Son de unos $15 \mathrm{~cm}$ de longitud total. Es abundante en la zona de estudio y por lo general se encuentra habitando escombros de coral muerto, son agresivos y territoriales. A los individuos pequeños se les ve merodeando las zonas de coral de fuego.

Mycteroperca intersticialis (cherna boca amarilla / yellowmouth grouper): su cuerpo está cubierto por manchas marrón y una coloración amarillo claro alrededor de la boca y al borde de la aleta dorsal. Alcanza $70 \mathrm{~cm}$ de longitud total. Esta especie es medianamente abundante en el área y se observa generalmente nadando algunos metros sobre el arrecife.

M. tigris (cherna tigre / tiger grouper): su coloración es marrón con nueve bandas blancas longitudinales sobre el dorso. Posee puntos marrón rojizo en la cabeza y el abdomen y las aletas anal, caudal y dorsal presentan manchas marrones irregulares. La parte interna de la boca es anaranjada. Es poco abundante en la zona y se le observa merodeando el arrecife.

Myripristis jacobus (pez ardilla / blackbar soldierfish): presenta una coloración rojo intenso y posee una raya negra detrás de la branquia. Alcanza $23 \mathrm{~cm}$ longitud total. También se presenta en la zona como una especie medianamente abundante.

Lacthophrys triqueter (pez cofre / smooth trunkfish): la parte alta del caparazón y la cola 
son negruzcos con numerosos puntos blancos. Presenta una coloración marrón detrás de las aletas pectorales y el abdomen, y todo el caparazón se encuentra recubierto por hexágonos claramente visibles de color blanco. Los labios y los ojos son negros. Son poco abundantes en la zona de Little Reef y se les observa merodeando cerca del fondo del arrecife.

Lutjanus analis (pargo chino / mutton snapper): es la especie más común dentro de este grupo. Su coloración es más o menos rojiza y presenta un punto negro lateral. Se observó de manera muy abundante y, por lo general, formando pequeños grupos dentro del arrecife.

L. jocu (pargo dientón / dog snapper): presenta una coloración marrón y tonos amarillo quemado en las aletas y el vientre, con marcadas barras verticales sobre el cuerpo. Por lo general posee un triangulo amarillo bajo el ojo. Su talla es de $30 \mathrm{~cm}$ de longitud total. Generalmente habita las zonas más someras del arrecife, formando cardúmenes y nadando de forma rápida e intermitente de un lado para otro dentro de las cabezas de coral. Los individuos más pequeños se observan cerca del fondo protegidos por parches coralinos. Son muy abundantes dentro del área de Little Reef.

Ocyurus chrysurus (saltona rabiamarilla / yellowtail snapper): posee una línea amarilla que va desde la parte anterior hasta la parte posterior, atravesando todo su cuerpo lateralmente, el resto del cuerpo es de color azul claro y la cola es amarilla. Es endémico del Atlántico occidental. Nada en grupos pequeños de 10 ejemplares. Asociado a arrecifes y zonas arenosas. Pelágico y filtrador. Esta especie, al igual que las dos anteriores, es muy abundante en el área de estudio.

Pomacanthus arcuatus (angel gris / gray angelfish): el juvenil posee cinco líneas amarillas que atraviesan su cuerpo sobre un fondo negro; a medida que crece estas líneas se tornan blancas hasta desaparecer en un fondo completamente gris típico del adulto, el cual presenta tonos entre gris claro y gris oscuro en todo su cuerpo. Su longitud total sobrepasa los $50 \mathrm{~cm}$. Al igual que los anteriores es poco abundante en el arrecife y se le observa solitario o en parejas.

$P$. paru (angel francés / french angelfish): su cuerpo es marrón oscuro con un dorado intenso al borde de las escamas cuando es adulto. El juvenil es negro con 4 bandas amarillas sobre el cuerpo y otra al rededor de la aleta caudal, el borde de la aleta anal es azul. Alcanza $40 \mathrm{~cm}$ de longitud total. Es medianamente abundante en el área de estudio y siempre se encuentra en parejas. Los juveniles presentan hábitos limpiadores.

Pseudopeneus maculatus (salmonete / spotted goatfish): presenta tres manchas oscuras bien notorias con intervalos regulares en la región lateral, la primera de ellas se encuentra por encima del opérculo. Alcanza los $30 \mathrm{~cm}$ longitud total. Al igual que la especie anterior es abundante dentro de la zona y habita los fondos arenosos que rodean el arrecife.

Seriola dumerili (jurel rayado o jurel ámbar / greater amberjack): se destaca por tener una línea longitudinal de color azul que atraviesa el ojo de la boca hasta el dorso. Estos individuos alcanzan una longitud total de casi $2 \mathrm{~m}$ y su carne es muy apetecida. En el área tan sólo fueron observados algunos individuos solitarios de tallas pequeñas que rondaban de lejos el arrecife.

Scarus coeruleus (loro azul / blue parrotfish): es de color azul pastel fuerte en estado adulto y azul claro con manchas salmón en la cabeza en estado juvenil. Los individuos un poco más desarrollados presentan una mancha amarilla en la cabeza. En machos adultos el hocico es protuberante. Pueden alcanzar una longitud total de $1.20 \mathrm{~m}$, aunque generalmente llegan a los $60 \mathrm{~cm}$. Los individuos de menor tamaño son especialmente abundantes en el arrecife de Little Reef y los de tallas grandes se presentan en menor grado.

S. guacamaia (loro reina / rainbow parrotfish): todo su cuerpo presenta colores amarillo, naranja y azul pastel que irradian iridiscencia. La aleta dorsal y pectoral son verdes y el resto anaranjadas. Los machos adultos presentan una coloración bronce en la parte anterior. Su talla es de $40 \mathrm{~cm}$. Son muy abundantes en el área de estudio y se les observa merodeando las praderas de pastos, algas y el arrecife. 
Revista Luna Azul - MACROFLORA Y MACROFAUNA ASOCIADAS AL COR... Página 12 de 17

Revista Luna Azul

No. 25, Julio - Diciembre 2007

Sparisoma viride (loro común / stoplight parrotfish): su nombre se debe a la coloración tan llamativa de su cuerpo. Presentan dicromatismo sexual, siendo el macho de color rojo oscuro con negro y la hembra de colores pastel. Alcanzan los $50 \mathrm{~cm}$ de longitud total. Son muy abundantes en la zona y, de igual manera que la especie anterior, merodean las áreas ocupadas por pastos, algas y arrecife de coral.

Sphyraena barracuda (barracuda / great barracuda): alcanza los $2 \mathrm{~m}$ de longitud total, posee unos caninos muy fuertes y en ciertos casos puede llegar a atacar al hombre. Su cuerpo es alargado y su coloración gris plateado. En la zona de Little Reef se presentan como individuos esporádicos de media agua que merodean el arrecife en busca de alimento.

Stegastes planifrons (damisela común / threespot damselfish): su coloración es simple, el cuerpo en su totalidad es marrón oscuro con visos amarillos. Tiene un punto oscuro en la base de la aleta pectoral y otro en la cola. Los juveniles son amarillos con un punto negro con azul sobre el dorso. Alcanza $10 \mathrm{~cm}$ de longitud total. Se caracterizan por ser los peces más agresivos de los arrecifes cuando se trata de defender su territorio. Son muy abundantes en Little Reef y siempre están rondando su territorio. Se les observa raspando el sustrato duro alimentándose de algas.

S. partitus (damisela bicolor / bicolor damselfish): de coloración muy variable. La característica más notoria es la división marcada entre el color marrón oscuro de la parte anterior y el color claro de la posterior. Puede presentar cambios de coloración con la edad, el sexo o durante el cortejo. Su talla es de $8 \mathrm{~cm}$ de longitud total. Al igual que la especie anterior es muy abundante en el área y se le observa en cardúmenes.

Thalasoma bifasciatum (cabeza azul / bluehead): se caracteriza porque la parte anterior del cuerpo es de color azul y la posterior amarillo-verdosa, delimitadas las dos porciones por un par de barras negras. Su talla máxima es de $12 \mathrm{~cm}$ de longitud total. Son abundantes en la zona y se les encuentra por todos lados del arrecife. Los juveniles son de hábitos limpiadores y nadan en grupo.

Trachinocephalus myops (pez lagarto / snake fish): presenta tonalidades de marrón y colores claros que le permiten camuflarse con el fondo. Como su nombre lo indica, tiene la apariencia de un lagarto y se arrastra sobre los fondos arenosos en busca de alimento o camuflaje. Son poco abundantes en la zona de estudio.

Tabla 1

Especies, hábitat, abundancia y registros previos de los organismos característicos del necton y bentos en el Cordón Arrecifal de Little Reef, en la Isla de San Andrés, Colombia. (1) Barriga-Bonilla et al. (1969), (2) Geister (1975); (3) Schnetter (1978); (4) Victoria-Daza y Gómez-Niño (1984); (5) Díaz-Merlano et al. (1995); (6) CORALINA (1997b); (*) Nuevos registros.

\begin{tabular}{|c|c|c|c|}
\hline $\begin{array}{c}\text { TAXA / FAMILIAS I } \\
\text { ESPECIES }\end{array}$ & HABITAT & ABUNDANCIA & \begin{tabular}{|c|} 
REGISTROS \\
PREVIOS \\
\end{tabular} \\
\hline \multicolumn{4}{|l|}{ ALGAS } \\
\hline \multicolumn{4}{|l|}{ Valoniaceae } \\
\hline Ventricaria ventricosa & Cabezas coral muerto & Poco abundante & 3 \\
\hline \multicolumn{4}{|l|}{ Caulerpaceae } \\
\hline Caulerpa racemosa & Coral vivo y muerto & Muy abundante & 3 \\
\hline \multicolumn{4}{|l|}{ Dictyotaceae } \\
\hline Dictyota spp. & Coral vivo y muerto & Muy abundante & $1,3,5$ \\
\hline \multicolumn{4}{|l|}{ Codiaceae } \\
\hline Halimeda opuntia & Fondo arenoso & Abundante & $1,3,5$ \\
\hline \multicolumn{4}{|l|}{ FANEROGAMAS } \\
\hline \multicolumn{4}{|l|}{ Thalassioideae } \\
\hline Thalassia testudinum & Fondo arenoso & Muy abundante & 1,5 \\
\hline \multicolumn{4}{|l|}{ Cymodoceoideae } \\
\hline Syringodium filiforme & Fondo arenoso & Poco abundante & 5 \\
\hline
\end{tabular}


Revista Luna Azul - MACROFLORA Y MACROFAUNA ASOCIADAS AL COR... Página 13 de 17

\begin{tabular}{|c|c|c|c|}
\hline CELENTERADOS & & & \\
\hline \multicolumn{4}{|l|}{ Actinidae } \\
\hline Condylactis gigantea & Coral, sustrato duro & Poco abundante & * \\
\hline \multicolumn{4}{|l|}{ Gorgonidae } \\
\hline Gorgonia ventalina & Sustrato duro arrecife & Esporádica & 1 \\
\hline \multicolumn{4}{|l|}{ Plexauridae } \\
\hline Plexaura cf. homomala & Sustrato duro arrecife & Poco abundante & $\star$ \\
\hline \multicolumn{4}{|l|}{ Milleporidae } \\
\hline Millepora complanata & Sustrato duro arrecife & Muy abundante & $1,2,5$ \\
\hline \multicolumn{4}{|l|}{ Diploridae } \\
\hline Diploria clivosa & Sustrato duro arrecife & Poco abundante & 2 \\
\hline Diploria strigosa & Sustrato duro arrecife & Muy abundante & 2,5 \\
\hline \multicolumn{4}{|l|}{ Poritidae } \\
\hline Porites astreoides & Sustrato duro arrecife & Abundante & 2 \\
\hline Porites porites & Fondo arenoso & Abundante & $1,2,5$ \\
\hline \multicolumn{4}{|l|}{ Siderastreidae } \\
\hline Siderastrea siderea & Sustrato duro arrecife & Poco abundante & 2,5 \\
\hline \multicolumn{4}{|l|}{ Faviidae } \\
\hline Montastrea annularis & Sustrato duro arrecife & Muy abundante & $1,2,5$ \\
\hline \multicolumn{4}{|l|}{ Agariciidae } \\
\hline Agaricia agaricites & Coral muerto & Poco abundante & $1,2,5$ \\
\hline \multicolumn{4}{|l|}{ Acroporidae } \\
\hline Acropora palmata & Sustrato duro arrecife & Poco abundante & $1,2,5$ \\
\hline \multicolumn{4}{|l|}{ Meandriidae } \\
\hline Meandrina meandrites & Sustrato duro arrecife & Esporádica & 1,2 \\
\hline \multicolumn{4}{|l|}{ POLIQUETOS } \\
\hline \multicolumn{4}{|l|}{ Sabellidae } \\
\hline Sabellastarte sp. & Cabezas coral & Muy abundante & $\star$ \\
\hline \multicolumn{4}{|l|}{ Serpullidae } \\
\hline Spirobranchus sp. & Cabezas coral & Muy abundante & $\star$ \\
\hline \multicolumn{4}{|l|}{ EQUINODERMOS } \\
\hline \multicolumn{4}{|l|}{ Stichopodidae } \\
\hline Isostichopus sp. & Fondo arenoso, pasto & Abundante & $\star$ \\
\hline \multicolumn{4}{|l|}{ Diadematidae } \\
\hline Diadema antillarum & Coral & Abundante & 1 \\
\hline \multicolumn{4}{|l|}{ Toxopneustidae } \\
\hline Tripneustes venticosus & Thalassia & Muy abundante & 5 \\
\hline \multicolumn{4}{|l|}{ Echinometridae } \\
\hline Echinometra lucunter & Fondo arrecifal & Esporádica & 5 \\
\hline \multicolumn{4}{|l|}{ CRUSTACEOS } \\
\hline \multicolumn{4}{|l|}{ Stenopodidae } \\
\hline Stenopus hispidus & Cuevas arrecifales & Poco abundante & $\star$ \\
\hline \multicolumn{4}{|c|}{\begin{tabular}{|l|l|} 
Majidae & \\
\end{tabular}} \\
\hline Stenorhynchus seticornis & Anémonas & Poco abundante & $\star$ \\
\hline Paniluridae & & & \\
\hline Panulirus argus & Grietas arrecifales & Esporádica & 6 \\
\hline$\overline{\text { PECES }}$ & & & \\
\hline Dasyatidae & & & \\
\hline Dasyatis sabina & Fondo arenoso & Poco abundante & $\star$ \\
\hline Ginglymostomidae & & & \\
\hline Ginglymostoma cirratum & Fondo arenoso & Esporádica & $\star$ \\
\hline
\end{tabular}


Revista Luna Azul - MACROFLORA Y MACROFAUNA ASOCIADAS AL COR... Página 14 de 17

Revista Luna Azul

No. 25, Julio - Diciembre 2007

\begin{tabular}{|c|c|c|c|}
\hline \multicolumn{4}{|l|}{ Holocentridae } \\
\hline Holocentrus adscensionis & Cuevas arrecifales & Abundante & 1 \\
\hline Holocentrus rufus & Cuevas, grietas & Medianamente $\mathrm{ab}$. & 1 \\
\hline Myripristis jacobus & Cuevas arrecifales & Medianamente $a b$. & 1 \\
\hline \multicolumn{4}{|l|}{ Fistularidae } \\
\hline Fistularia tabacaria & Thalassia y arrecife & Poco abundante & * \\
\hline \multicolumn{4}{|c|}{\begin{tabular}{|l|l|} 
Serranidae & \\
\end{tabular}} \\
\hline Epinephelus ascensionis & Grietas arrecifales & Poco abundante & 1 \\
\hline Epinephelus guttatus & Fondo arrecifal & Abundante & 6 \\
\hline Hypoplectrus puella & Fondo arrecifal & Poco abundante & $\star$ \\
\hline $\begin{array}{l}\text { Mycteroperca } \\
\text { intersticialis }\end{array}$ & Sobre el arrecife & Medianamente ab. & 4 \\
\hline Mycteroperca tigris & Media agua & Poco abundante & 4,6 \\
\hline \multicolumn{4}{|l|}{ Carangidae } \\
\hline Caranx hippos & Media agua & Esporádica & $\star$ \\
\hline Caranx ruber & Media agua & Poco abundante & $\star$ \\
\hline Seriola dumerili & Media agua & Esporádica & $\star$ \\
\hline \multicolumn{4}{|l|}{ Lutjanidae } \\
\hline Lutjanus analis & Media agua & Muy abundante & 6 \\
\hline Lutjanus jocu & Media agua & Muy abundante & 6 \\
\hline Ocyurus chrysurus & Media agua & Muy abundante & 1,6 \\
\hline \multicolumn{4}{|l|}{ Haemulidae } \\
\hline Haemulon aurolineatum & Media agua y fondo & Muy abundante & 1 \\
\hline Haemulon chrysargirum & Media agua y fondo & Muy abundante & 1 \\
\hline Haemulon flavolineatum & Media agua y fondo & Muy abundante & 1 \\
\hline Haemulon plumieri & Media agua y fondo & Abundante & 6 \\
\hline Haemulon sciurus & Media agua y fondo & Abundante & 6 \\
\hline \multicolumn{4}{|l|}{ Mullidae } \\
\hline Mulloidichtys martinicus & Arena y Thalassia & Abundante & 1,6 \\
\hline $\begin{array}{l}\text { Pseudopeneus } \\
\text { maculatus }\end{array}$ & Fondo arenoso & Abundante & 1 \\
\hline \multicolumn{4}{|l|}{ Chaetodontidae } \\
\hline Chaetodon capistratus & Fondo arrecifal & Abundante & 1 \\
\hline Chaetodon ocellatus & Fondo arrecifal & Abundante & $\star$ \\
\hline Chaetodon striatus & Fondo arrecifal & Abundante & 1 \\
\hline \multicolumn{4}{|l|}{ Pomacantidae } \\
\hline Holocanthus ciliaris & Fondo arrecifal & Poco abundante & 1 \\
\hline Holocanthus tricolor & Fondo arrecifal & Poco abundante & 1 \\
\hline Pomacanthus arcuatus & Fondo arrecifal & Poco abundante & 1 \\
\hline Pomacanthus paru & Fondo arrecifal & Medianamente $a b$. & 1 \\
\hline \multicolumn{4}{|l|}{ Pomacentridae } \\
\hline Abudefduf saxatilis & Fondo arrecifal & Muy abundante & 1 \\
\hline Chromis cyaneus & Fondo arrecifal & Muy abundante & $\star$ \\
\hline $\begin{array}{l}\text { Mycrospathodon } \\
\text { chrysurus }\end{array}$ & Coral muerto y vivo & Abundante & 1 \\
\hline Stegastes partitus & Fondo arrecifal & Muy abundante & 1 \\
\hline Stegastes planifrons & Fondo arrecifal & Muy abundante & 1 \\
\hline \multicolumn{4}{|l|}{ Labridae } \\
\hline Bodianus rufus & Fondo arrecifal & Medianamente ab. & $\star$ \\
\hline Thalassoma bifasciatum & Fondo arrecifal & Abundante & 1 \\
\hline \multicolumn{4}{|l|}{ Scaridae } \\
\hline Scarus caeruleus & Fondo arrecifal & Abundante & 1 \\
\hline Scarus guacamaia & Pastos, algas, coral & Muy abundante & $\star$ \\
\hline Sparisoma viride & Pastos, algas, coral & Muy abundante & 1 \\
\hline \multicolumn{4}{|l|}{ Gobiidae } \\
\hline Gobiosoma genie & Cabezas de coral & Esporádica & $\star$ \\
\hline
\end{tabular}


Revista Luna Azul - MACROFLORA Y MACROFAUNA ASOCIADAS AL COR... Página 15 de 17

\begin{tabular}{|l|l|l|l|} 
Gobiosoma oceanops & Cabezas coral cerebro & Poco abundante & \multirow{2}{*|}{} \\
\hline Acanthuridae & & & \\
\hline Acanthurus chirurgus & Media agua y fondo & Muy abundante & 1,6 \\
\hline Acanthurus coeruleus & Media agua & Muy abundante & 1 \\
\hline Sphyraenidae & & & \\
\hline Sphyraena barracuda & Media agua & Esporádica & 1,6 \\
\hline Synodontidae & & & \\
\hline Trachinocephalus myops & Fondo arenoso & Poco abundante & $*$ \\
\hline Ostracidae & & & \\
\hline Lacthophrys triqueter & Media agua y fondo & Poco abundante & 1,4 \\
\hline Diodontidae & & & \\
\hline Diodon histrix & Arena, grietas, cabezas & Poco abundante & \\
\hline
\end{tabular}

\section{DISCUSIÓN Y CONCLUSIONES}

Díaz-Merlano et al. (1995) proponen para la zona de Little Reef cuatro tipos de arrecifes de parche según el grado de turbulencia al que se encuentran expuestos, a la profundidad y a la composición de las especies: (1) Tipo A.palmata-D.strigosaM.complanata, ubicados en las áreas más expuestas al oleaje de la laguna y cuyas crestas alcanzan la superficie; (2) Tipo M. annularis o parches aislados de cabezas coralinas, ubicados en las áreas centrales de la laguna y cuya parte superior no alcanza la superficie; (3) Tipo cervicornis, que hoy en día se encuentran prácticamente destruidos; y (4) Tipo P. furcata, ubicados en las áreas más someras y calmadas. Por lo que de acuerdo a esta clasificación definen el extremo oeste y noroeste de Little Reef como una formación de tipo $M$. annularis y los demás parches como una de tipo A.palmata-D.strigosa-M.complanata. Estas observaciones concuerdan con las realizadas para este estudio en donde las especies dominantes al centro y este del arrecife son $D$. strigosa, $M$. complanata y $A$. palmata, aunque esta última presenta un alto grado de mortalidad en el área; y al norte, oeste y centro se presenta una gran abundancia de $M$. annularis y $D$. strigosa.

Se observa una gran proliferación de algas sobre el arrecife de Little Reef, las cuales han establecido una clara competencia por el espacio, causando mortalidad y estrés en las especies de coral que habitan el área. Según Díaz-Merlano et al. (1995) el crecimiento de algas sobre corales en decadencia puede contribuir a precipitar su muerte. Este crecimiento acelerado de algas puede deberse al incremento en el contenido de nutrientes provenientes de los desechos domésticos que son arrojados en el área, lo cual contribuye junto con el incremento de la sedimentación, la aparición de sustancias tóxicas (detergentes y blanqueadores), y otros agentes, con el deterioro del coral. En San Andrés, la contaminación por aguas negras se produce de dos formas: por descarga del alcantarillado urbano al mar sin tratamiento alguno y por rebose de pozos sépticos. Melendro (1990) mencionó niveles altos de nutrientes alrededor de la isla, citando que fueron inferidos por $\mathrm{H}$. von Prahl (com. pers.) a partir del crecimiento excesivo de algas.

Se presenta una gran mortalidad de corales en la zona de Little Reef y, en general, en la Isla de San Andrés, lo cual puede deberse en parte a las causas anteriormente mencionadas, además de otros factores como la aparición de enfermedades y el blanqueamiento o expulsión masiva de algas simbióticas debido al sobrecalentamiento de las aguas. En Little Reef se observa claramente la presencia de bandas blancas y blanquamiento en las diferentes zonas con predominio de Acropora, y de banda negra sobre las especies semiesféricas. Geister (1973) registró, para algunas áreas poco profundas al oriente de la isla, temperaturas en el agua que podían sobrepasar los 40 oC, en días de calma y baja mar. Aunque según Díaz-Merlano et al. (1995) las comunidades de las áreas donde este sobrecalentamiento es usual, han logrado adaptarse y soportar altas temperaturas, además de haber adquirido una alta capacidad de recuperación. Sin embargo, cambios bruscos en la temperatura del agua, pueden generar sobre estas comunidades una considerable mortalidad coralina, como las que se han registrado en general para todo el Caribe, incluyendo San Andrés entre 1986 y 1988, afectando las colonias de corales más sensibles como Montastrea, Siderastrea y Porites (Melendro, 1990; INDERENA, 1992). En cuanto a las enfermedades citadas, 
Garzón-Ferreira y Cano-Correa (1991), atribuyen a la enfermedad de banda blanca las dramáticas mortandades de Acropora palmata, A. cervicornis y Agaricia tenuifolia. Siendo esta enfermedad de origen bacteriano la posible responsable de la extensiva mortalidad de A. palmata en San Andrés (Geister, 1975; Díaz-Merlano et al., 1995). Este último registra para la zona norte de la laguna, incluyendo Little Reef, un 50 al 95\% de mortalidad de A. palmata a causa de esta enfermedad. Por otro lado, la enfermedad de banda negra, causada por la cianobacteria Phormidium corallyticum, que ataca principalmente cabezas coralinas de Siderastrea, Diploria y Montastrea (Rutzel et al., 1983), ha sido observada en el área de estudio y es según Díaz-Merlano et al. (1995), la probable responsable de la mortalidad parcial detectada en las especies coralinas semiesféricas en la isla de San Andrés.

Al comparar los resultados obtenidos en trabajos anteriores (Barriga-Bonilla et al., 1969; Geister, 1973; Díaz-Merlano et al. (1995), es evidente que por los objetivos y el tiempo invertido en dichas investigaciones, el inventario de flora y fauna fueron diferentes, lo cual se evidencia en el número de registros de la presente contribución.

\section{AGRADECIMIENTOS}

A la Escuela de Buceo Styngray y a su instructor Iván Velázquez, a Johanna Gaviria y a Juan Pablo.

\section{BIBLIOGRAFÍA}

- BARRIGA-BONILLA, E., J. I. HERNÁNDEZ-CAMACHO, I. JARAMILLO, R. JARAMILLO-MEJÍA, L. M. MORA-OSEJO, P. PINTO y P. M. RUÍZ-CARRANZA. 1969. La Isla de San Andrés. Contribuciones al conocimiento de su ecología, flora, fauna y pesca. Public. Universidad Nacional de Colombia. Bogotá D.E. (Colombia), $152 \mathrm{p}$.

- BOER, B. DE, D. HOOGERWERF, I. KRISTENSEN y J. POST. 1973. Antillean fish guide. Stinapa, 7: 1-109.

- BORRERO, J. M. y L. GONZÁLEZ. 1983. Investigación ecológica y gestión ambiental en las Islas de San Andrés y Providencia, pp. 11-22 In: Borrero, J. M. y L. González. Investigación Ecológica y Gestión Ambiental en las Islas de San Andrés y Providencia. Fund. Invest. Protec. Medio Ambiente (FIPMA). Cali. (Colombia), $288 \mathrm{p}$.

- BOOlotian, R. A. 1975. Biología de los atolones de coral. BSCS/CECSA/CNEB, A.C. México D. F. (México).

- COLIN, P. 1978. Caribbean reef invertebrates and plants. T.F.H. Publications. London (U. K.), 512 p.

- CONTRERAS, R. 1983. Evaluación preliminar del estado de los ecosistemas costeros en la Isla de San Andrés (Atlántico colombiano) con especial énfasis en la zona de Bahía Hooker, pp. 81-103 In: Borrero, J. M. y L. González. Investigación Ecológica y Gestión Ambiental en las Islas de San Andrés y Providencia. Fund. Invest. Protec. Medio Amb. (FIPMA), Cali. (Colombia), 288 p.

- CORALINA. 1997a. Arrecifes coralinos. San Andrés, Providencia y Santa Catalina nuestra reserva de biosfera. Cartilla del Proyecto de Manejo y Conservación de Recursos Marinos y Pesqueros. San Andrés Isla. (Colombia), 16 p.

- 1997b. La pesca artesanal. San Andrés, Providencia y Santa Catalina nuestra reserva de biosfera. Cartilla del Proyecto de Manejo y Conservación de Recursos Marinos y Pesqueros. San Andrés Isla. (Colombia), 35 p.

- DÍAZ-MERLANO, J. M., J. GARZÓN-FERRÉIRA y S. ZEA. 1995. Los arrecifes coralinos de la Isla de San Andrés, Colombia: Estado actual y perspectivas para su conservación. Academia Nacional de Ciencias Exactas, Físicas y Naturales. Colección Jorge Alvarez Lleras No 7. Santa Fe de Bogotá. (Colombia), 150 p.

- GARZÓN-FERREIRA, J. y M. CANO-CORREA. 1991. Tipos, distribución, extensión y estado de conservación de los ecosistemas marinos costeros del Parque Nacional Natural Tayrona. Manuscrito VII Concurso Nac. Ecol. FEN Colombia. Santa Fe de Bogotá D. C. (Colombia), 82 p.

- Geister, J. 1973. Los arrecifes de la Isla de San Andrés (Mar Caribe, Colombia). Mitt. Inst. Colombo-Alemán Investigaciones Científicas, 7: 211-228. 
Revista Luna Azul - MACROFLORA Y MACROFAUNA ASOCIADAS AL COR... Página 17 de 17

- 1975. Riffbau und geologische entwicklungsgeschichte der Insel San Andrés (Westliches Karibisches Meer, Kolumbien). Stuttugarter Beitr. Naturk. Serie B (Geologie und Paläontologie). Stuttgart, nr. 15, 203 p.

- 1976. Los arrecifes de barlovento al norte de la Isla de San Andrés (Mar Caribe, Colombia). Mem. 1er. Congreso Colombiano de Geología, Bogotá, 1969: 173-186.

- GÓMEZ-ÑIÑO, D. P. y P. VICTORIA-DAZA. 1980. Inventario preliminar de los peces de la Isla de San Andrés y noroeste de la Isla de Providencia (Mar Caribe de Colombia). Tesis Profesional. Fac. Biol. Marina. Univ. de Bogotá Jorge Tadeo Lozano, 198 p.

- 1986. Peces de la Isla de San Andrés y noroeste de la Isla de $\overline{\text { Providen }}$ cia (Mar Caribe de Colombia) inventario en arrecifes coralinos, praderas marinas y aguas costeras. UBJTL-Bol. Ecotrópica, 13: 41-85.

- GREENBERG, I. 1986. Guide to corals and fishes of Florida, the Bahamas and the Caribbean. IOF (An International Oceanographic Foundation Selection). Miami (Florida) USA, $65 \mathrm{p}$.

- INDERENA. 1992. Diagnóstico preliminar sobre los problemas ambientales en San Andrés Isla. San Andrés Isla (Colombia). Instituto Nacional de los Recursos Naturales Renovables y del Ambiente. Bogotá D. E. Inf. Técnico, 10 p.

- KAPRAUM, D. F. 1972. Notes on the benthic marine algae of San Andrés, Colombia. Carib. J. Sci., 12 (3-4):199-203.

- MELENDRO, E. 1990. Informe de comisión San Andrés y Providencia, Islas. INDERENA- Sec. Evaluac. Ambient. Bogotá D. E. Inf. Técnico, 12 p.

- PFLUEGER, A. y R. SMILEY. 1974. Fisherman's handbook. Windward. Florida. (USA), $94 \mathrm{p}$.

- PRAHL, H. VON. y H. ERHARDT. 1985. Corales y arrecifes coralinos. FENColombia. Bogotá D. E. (Colombia), 295 p.

- RUTZEL, K., D. L. SANTAVY y A. ANTONIUS. 1983. The black band disease of the Atlantic coral reef. III. Distribution, Ecology and Development. Marine Ecology Progress Series, 4 (4): 329-358.

- SCHNETTER, R. 1978. Marine algae der Karibischen kusten von Kolumbien. II. Chlorophyceae. Bibliotheca Phycologica, 42: 1-99.

- STOKES, F. J. 1980. Coral reef fishes of the Caribbean and adjacent tropical waters including Florida, Bermuda and the Bahamas. Lippincott y Crowell, New York (USA), $160 \mathrm{p}$.

- VICTORIA-DAZA, P. y D. GÓMEZ-ÑIÑO. 1984. Nuevos registros de peces para la Isla de San Andrés (Mar Caribe de Colombia). Anales Instituto de Investigaciones Marinas, Punta de Betín, 14:115-132. 